\title{
La gestion des eaux pluviales en ville: regard croisés sur Paris et Rio de Janeiro
}

\author{
Ana Lucia Britto, Bernard Barraqué*
}

Resumé Les eaux pluviales urbaines font partie des éléments naturels qui peuvent représenter à la fois une ressource et une menace pour la vie urbaine. Dans les métropoles françaises et brésiliennes, ce thème est désormais au cœur de la planification urbaine et des projets urbains. En partant de l'approche de la gestion durable des eaux en ville qui intégre urbanisme et cicle urbain de l'eau, on cherche à indiquer les potentialités et les limites des instruments de planification concernant la gestion des eaux pluviales urbaines à travers des regards croisés entre Paris et Rio de Janeiro.

Mots-clefs: gestion des eaux pluviales, Rio de Janeiro, Paris.

\section{Águas pluviais urbanas : olhares cruzados sobre o seu planejamento em Paris e no Rio de Janeiro}

Resumo O texto trata do manejo de águas pluviais urbanas entendendo que esses elementos da natureza na cidade podem representar em recurso ou uma ameaça para a vida urbana, sendo essa temática central no planejamento e nas ações de urbanismo. São abordadas as cidades de Paris e Rio de Janeiro, analisando os instrumentos de planejamento urbano e de planejamento da gestão e manejo de águas pluviais adotados, através de um olhar cruzado entre as duas cidades, indicando potencialidades e limites desses instrumentos. A lente da análise são as perspectivas apontadas pela drenagem sustentável e pelo WSUD (Water Sensitive Urban Design/ Urbanismo Sensível à Água), que trazem a possibilidade que um planejamento urbano que integre o ciclo urbano da água.

Palavras-chave: águas pluviais urbanas, Rio de Janeiro, Paris.

\section{Urban stormwater management : pers- pectives from Paris and Rio de Janeiro}

\begin{abstract}
Urban stormwater is one of the natural elements that can be both a resource and a threat to urban life. In French and Brazilian cities, this theme is now at the heart of urban planning and urban design. Starting from the approach of the water sensitive urban design which integrates urban planning and design and the urban cicle of the water, we seeks to indicate the potentialities and the limits of planning instruments concerning the urban rainwater management through crossed views between Paris and Rio de Janeiro.
\end{abstract}

Keywords: urban stormwater management, Rio de Janeiro, Paris. 
a nature en ville est entendue dans cet texte a partir de l'element eau, en considerant

- le cycle d'eau en ville, et en particulier les eaux pluviales. Les eaux pluviales urbaines font partie des éléments naturels qui peuvent représenter à la fois une ressource et une menace pour la vie urbaine. En ville, entre les trottoirs, les toitures, les parkings et les routes, les zones imperméables sont très nombreuses l'eau de pluie ruisselle avant d'être prise en charge par des réseaux de tuyaux. Dans les métropoles françaises et brésiliennes, ce thème est désormais au cœur de la planification urbaine et des projets urbains. On va le voir ici avec les villes de Paris et de Rio de Janeiro. L'objectif est d'analyser les instruments de planification adoptés pour la gestion des eaux pluviales, à travers des regards croisés entre les deux villes. On cherche à questionner la portée de l'action publique dans ce domaine et d'indiquer les potentialités et les limites des instruments concernant la gestion des eaux pluviales. Notre angle d'analyse s'appuie sur la notion d'assainissement urbain durable et par ce qu'on appelle en anglais WSUD (Water Sensitive Urban Design). Dans la première partie seront présentées de façon brève les perspectives conceptuelles qui orientent l'analyse ; dans la deuxième partie le cadre réglementaire pour l'assainissement et la gestion de l'assainissement des eaux de pluie en France et au Brésil; dans la troisième partie les cas de Paris et de Rio de Janeiro, et leurs instruments de planification, en essayant d'établir un regard croisé entre les deux villes. Dans les conclusions, nous cherchons à fournir des éléments pour la réflexion proposée par le séminaire sur l'eau de pluie, en tant que "Janus" avec le visage du risque et le visage d'un atout potentiel de développement urbain intégré avec la nature.

\section{Les eaux pluviales en ville: d'une menace à une ressource}

La plupart des urbanistes et ingénieurs qui travaillent aujourd'hui sur l'eau en ville ont adopté concept le concept de Sustainable Urban Water Management (SUWM) qui est associé dans le cadre de projets urbains à celui de WSUD. Les deux concepts proviennent d'une évolution dans la gestion du drainage urbain et du cycle urbain de l'eau qui s'est produit au cours des dernières décennies, passant d'approches très ciblées (généralement dans le seul but de réduire les inondations) à une approche à plusieurs objectifs, liées à l'aménagement urbain (Fletcher et al, 2015). Ces objectifs concernent la gestion et la planification, ainsi que les projets urbains. On envisage

* Ana Lucia Britto est Géographe, Professeur de la Faculdade de Arquitetura e Urbanismo et du PROURB - Programa de Pós-graduação em Urbanismo da Universidade Federal do Rio de Janeiro, Brésil, ORCID <https://orcid.org/00000001-6558-8405>. Bernard Barraqué est Ingénieurs en Génie Civil, Professeur du Centre National de la Recherche Scientifique, ORCID <https://orcid.org/00000003-4638-3708> une conception qui vise à intégrer le cycle de l'eau, element central de la nature à la ville en s'interrogeant sur les fonctions multiples que les eaux de pluie peuvent remplir dans un contexte urbain. On passe d'une vision des eaux de pluie comme un problème ou une menace, à une vision de l'eau de pluie comme une ressource et un atout pour les projets d'urbanisme. En témoigne le colloque organisé par le SIAAP en mai 2006 : la pluie en ville, nuisance ou ressource ${ }^{1}$.

Le concept de projet urbain attentif à l'eau - WSUD a été initialement développé en Australie. WSUD est défini comme une coopération interdisciplinaire dans la gestion de l'eau, réunissant le génie de l'eau, I'urbanisme, le design urbain et le paysagisme, dans 
1 SIAAP (Syndicat Interdépartemental de I'Assainissement de l'Agglomération Parisienne): actes du colloque du 11 mai 2006. <https://www.oieau. fr/eaudoc/system/files/documents/42/210415/210415_doc. $\mathrm{pdf}>$. la prise en compte de toutes les parties du cycle hydrologique urbain ; on cherche à combiner la fonction de gestion de l'eau et des approches de design urbain et faciliter les synergies pour la durabilité sociale et culturelle (Langenbach et al., 2008; Hoyer et al., 2011)

Selon Wong (2007), dans le cadre du WSUD le terme water sensitive définit un nouveau paradigme dans la gestion intégrée du cycle hydrologique urbain, qui amène les différentes disciplines ci-dessus à réunir différents domaines de la gestion de l'eau allant de la fourniture de services dieau à la protection des milieux aquatiques dans les zones urbaines. L'auteur affirme que dans cette approche les valeurs communautaires et les demandes des zones urbaines guident nécessairement les décisions d`urbanisme et les pratiques de gestion de lyeau dans le but minimiser les impacts hydrologiques du développement urbain sur lsenvironnement (Wong, 2006). La gestion des eaux pluviales est un sous-ensemble du WSUD visant à contrôler les inondations, à gérer les débits, à améliorer la qualité de lseau et à exploiter les eaux pluviales pour compléter lıeau de distribution à des fins non potables (Lloyd \& al, 2002, apud Fletcher \& al, 2015).

Le terme anglais Sustainable Urban Water Management (SUWM) met l'accent sur la partie pluviale de la gestion durable de l'eau urbaine. Cette dernière propose de traiter ensemble les quatre dimensions du développement durable, à savoir les ' $3 \mathrm{E}$ ' (environnement, économie, équité) plus la gouvernance, dans une perspective où des solutions fondées sur la nature sont réhabilitées par rapport au tropisme précédent tourné vers la technologie (Barraqué \& al, 2015). En France, cette préoccupation a été confortée dès la fin des années 1970 par un programme de recherches et d'innovation du Plan Urbain, L'eau dans la Ville (Lelong, 1995)

En français le terme correspondant est la gestion durable des eaux pluviales. Dans un modelé de gestion durable des eaux pluviales, l'eau de ruissellement est considérée comme une ressource et non une nuisance. Plutôt que de tenter de les évacuer le plus rapidement possible, il faut favoriser le maintien des eaux sur le site, un écoulement plus lent et des méthodes de rétention et de décontamination avant leur arrivée dans les milieux hydriques. A travers des actions d'aménagement on se place dans une perspective d'atténuation du risque ou de réduction de la vulnérabilité de la ville aux inondations.

Les solutions techniques proposées et les alternatives pour la gestion des eaux pluviales sont les mêmes: la décentralisation des solutions et de la gestion, cherchant, autant que possible, à les intégrer dans le cycle de l'eau urbain et les conditions environnementales de l'environnement dans l'aménagement urbain; la fourniture, si possible, d'avantages esthétiques et paysagers des aménagements; la facilité et le faible coût de maintenance; la possibilité d'adaptation (aux événements extrêmes et aux changements futurs possibles dans le contexte dans lequel ils sont insérés); la multifonctionnalité et l'implication et l'acceptation de la population (Chocat, 2008 ; Hoyer \& al, 2011).

\section{Le cadre réglementaire pour la gestion des eaux de pluviales en France et au Brésil}

Aussi bien en France qu'au Brésil l'assainissement des eaux pluviales est de compétence des communes et collectivités territoriales. En France, Loi de modernisation de l'action 
publique territoriale et d'affirmation des métropoles (MAPTAM) du 27 janvier 2014 établit I'attribution aux intercommunalités d'une compétence ciblée et obligatoire relative à la gestion de l'eau, des milieux aquatiques et à la prévention des inondations (GEMAPI). Plus récemment, la Loi portant sur la Nouvelle Organisation Territoriale de la République (NOTRe) du 7 août 2015 met en place un regroupement intercommunal sans précédent, et fait obligation aux nouvelles communautés d'agglomération et communautés de communes, de prendre la compétence assainissement dans sa globalité.

Au Brésil la Loi 11.445/2007 définit les services de "saneamento básico" (approvisionnement en eau; collecte et traitement des égouts; drainage et gestion des eaux pluviales; nettoyage urbain et gestion des déchets solides). L'article définissant les services urbains de drainage et de gestion des eaux pluviales a été modifié en 2016 (Loi 13.308/2016). La nouvelle rédaction y inclut le drainage et la gestion des eaux pluviales, le nettoyage et l'inspection préventive des réseaux urbains dédiés à ensemble des activités, infrastructures et équipements opérationnels pour la collecte et le transport des eaux de pluie, mais aussi les dispositifs de rétention pour l'écrêtement des débits, puis le traitement et l'élimination finale des eaux pluviales dans les zones urbaines. Les services de saneamento básico sont de competence municipale, mais dans les régions métropolitaines la gestion de ces services doit être assurée par une sorte de syndicat mixte entre les communes membres et l'Etat fédéré.

En France, la législation nationale sur la gestion des eaux pluviales, est dispersée dans plusieurs codes et en définitive peu contraignante. Mais dès les années 1970, la méthode de calcul pour le dimensionnement des réseaux d'égout séparatifs, officielle depuis 1949, a été remise en cause dans les villes nouvelles où elle aurait conduit à des dimensionnements considérables et infinançables (Chatzis \& Dupuy, 2000). La gestion quantitative et la rétention des eaux de pluie ont été développées empiriquement dans des zones urbaines denses et peu pentues (Seine St Denis, Bordeaux, Lyon, Marseille). La Loi sur I'Eau (2006) spécifie que les aménagements doivent limiter l'imperméabilisation des sols et ne pas aggraver le risque d'inondation, et qui soumet les rejets importants d'eaux pluviales à une procédure "au titre de la loi sur l'eau". L'article L 421-6 et les articles R 111-2, R 111-8 et R 111-15 du Règlement National de l'Urbanisme, permettent soit d'imposer des prescriptions en matière de gestion des eaux soit de refuser une demande de permis de construire ou d'autorisation de lotir en raison d'une prise en compte insuffisante de la gestion de ces eaux dans le projet.

Au niveau local la collectivité territoriale compétente peut refuser le raccordement des eaux pluviales aux réseaux d'assainissement (unitaires ou séparatifs). L'Article L1331-1 du Code de la Santé Publique donne la possibilité à la commune de fixer des prescriptions pour le raccordement des eaux pluviales, dans le cadre de son pouvoir de police : la commune peut limiter, voire interdire les rejets sur voies publiques par arrêté municipal. Ces prescriptions peuvent, par exemple, porter sur des principes d'infiltration des eaux pluviales avant raccordement ou sur des valeurs limites de débits acceptables dans les réseaux. Le cas échéant, elles intègrent les orientations et règles définies dans le cadre du zonage pluvial de la collectivité.

Toujours au niveau local le Code Général des Collectivités Territoriales, spécifie que les communes doivent délimiter: 
" $3^{\circ}$ Les zones où des mesures doivent être prises pour limiter l'imperméabilisation des sols et pour assurer la maîtrise du débit et de l'écoulement des eaux pluviales et de ruissellement";

"4늘 zones où il est nécessaire de prévoir des installations pour assurer la collecte, le stockage éventuel et, en tant que de besoin, le traitement des eaux pluviales et de ruissellement lorsque la pollution qu'elles apportent au milieu aquatique risque de nuire gravement à l'efficacité des dispositifs d'assainissement".

Les mesures contraignantes à respecter sur un territoire donné sont précisées dans les documents d'urbanisme locaux tels que les SCOT, PLUi et Cartes communales, qui doivent prendre en compte les prescriptions des SDAGE et des SAGE, outils de planification de la ressource en eau. Le SDAGE (Schéma Directeur d'Aménagement et de Gestion des Eaux) est un outil de planification formulé par les organismes de bassin. Chacun des six grands bassins hydrographiques français, ainsi que les départements d'outre mer, disposent d'un SDAGE. II fixe pour une période de six ans les grandes orientations d'une gestion équilibrée de la ressource en eau et les objectifs à atteindre en termes de qualité et de quantité des eaux. Le SAGE (Schéma Directeur d'Aménagement et de Gestion des Eaux), décline à l'échelle d'une unité hydrographique ou d'un système aquifère. Le SCOT (Schéma de Cohérence Territoriale) est un document de planification à long terme, visant à fixer les objectifs du développement durable d'un territoire intercommunal. PLU OU PLUi Plan Local d'Urbanisme ou Plan Local d'Urbanisme Intercommunal est l'outil permettant d'exprimer le projet urbain de la commune ou de l'intercommunalité. II prend en compte les problématiques environnementales, dont la prévention du risque d'inondations par ruissellement pluvial et la préservation des milieux naturels.

Un autre instrument important pour la gestion des inondations par les collectivités territoriales est le Plan de Prévention des Risques d'Inondation (PPRI), bien qu'il ne concerne pas particulièrement les inondations liées au pluvial urbain. Le PPRI a été rendu obligatoire par la loi (n. 87-565 du 22 juillet 1987 relative à I'organisation de la sécurité civile et à la prévention des risques majeurs). Face au peu d'empressement de nombreuses communes à faire ces plans, prévus dès un autre texte de 1982, cette loi confiait la réalisation de tels PPRI par les préfets. La loi MAPTAM, mentionnée ci-dessus a re-décentralisé une partie de la gestion du risque (gestion des milieux aquatiques, des risques d'inondation et des "risques littoraux") vers les territoires concernés (par les risques d'inondation et de submersion marine) aux établissements publics de coopération intercommunale disposant d'une fiscalité propre (EPCI-FP). Elle détermine ainsi le transfert à leur endroit de l'aménagement d'un bassin, I'entretien et l'aménagement d'un cours d'eau, d'un canal, d'un lac, la protection contre les inondations d'un cours d'eau ou de la mer, la protection et la restauration de milieux aquatiques. Ces opérations relevaient jusque-là de plusieurs échelons territoriaux. Pour financer leurs actions, les intercommunalités peuvent instaurer une nouvelle taxe, plafonnée à 40 euros par an et par habitant. Le pari est fait que la compétence Gemapi va permettre d'harmoniser les documents d'urbanisme et ceux du risque d'inondation, comme le préconise la directive européenne sur ces risques d'inondations (CE 2007/60).

Au Brésil, le cadre règlementaire est encore moins précis qu'en France. La Constitution brésilienne de 1988 a institué l'obligation aux municipalités dont la population était 
2 Les zones spéciales d'intérêt social (ZEIS) sont des zones délimitées sur le territoire d'une ville, pour le logement des populations à faibles revenues. Elles doivent être prévus dans le plan directeur et délimités dans la loi de zonage. II peut s'agir de zones déjà occupées par des établissements précaires (comme les favelas) ou des terrains vides. Dans le premier cas, l'objectif est de flexibiliser les paramètres urbanistiques à travers un plan d'urbanisation spécifique visant la régularisation foncière. Dans le cas des zones vides, l'objectif est d'augmenter l'offre de terrains pour le logement social et de réduire son coût.

3 Les Plans Directeurs brésiliens sont I'équivalent du PLU en France. Ils on la force de loi mais doivent être complémentés par les Lois d'usage et occupation du sol. de plus de vingt mille habitants, de promulguer un plan directeur de développement urbain (article 182, § 1). Le Statut de la Ville, loi fédérale $n^{\circ} 10.257 / 01$, a déterminé le délai maximum pour l'élaboration du Schéma directeur de développement urbain, sa validité (10 ans), son renouvellement et son caractère participatif. Le contenu précis du Plan Directeur n'est pas établi de façon détaillée dans la loi nationale, mais elle met l'accent sur son objectif : assurer la fonction sociale de la ville et de la propriété foncière urbaine, de manière à garantir à tous les segments sociaux un accès à des terrains urbanisés et régularisés, à garantir le droit universel au logement et aux services urbains, ainsi qu'à mettre en œuvre des procédures de démocratie participative ; les instruments à employer sont définis dans le Statut de la ville, qui lui-même s'est basé sur des processus de gestion innovants dans les municipalités (Santos Junior et al, 2011).

Certains Etats fédérés ont édictés des lois sur le développement urbain qui établissent le contenu minimal des Plans directeurs de développement urbain municipaux. On peut dire que ce contenu est le suivant: (i) la délimitation des zones urbaines, des zones d'expansion urbaine et des zones l'urbanisation spécifique (ZEIS)2; (ii) le macrozonage (iii) l'indice d'occupation du sol (IOS) et le coefficient d'occupation du sol (COS) minimum et maximum, le bandes non constructibles, retraits avant, latéraux et arrière des bâtiments (iv) la délimitation des espaces verts dont la végétation doit être préservée.

Une étude de 2011 sur les Plans Directeurs de développement urbain municipaux montre que, par rapport à l'assainissement et la gestion des eaux de pluie, les lignes directrices établies dans ces documents sont très générales et les objectifs et les cibles ne sont pas clairement définis. Seuls quelques Plans Directeurs présentent des directives concernant d'élaboration de plans pour les services de saneamento básico (Britto, 2011). Pourtant, depuis la Loi $n^{\circ} 11.445 / 2007$ I'élaboration de ces plans est obligatoire, et ils doivent respecter un contenu minimal.

La municipalité à la possibilité d'élaborer des plans séparément (un plan pour chaque service) ou de façon unifiée (un seul plan pour les quatre services d'eau potable, d'assainissement, du pluvial et des déchets). Le plan a force de loi, et sa révision doit se faire tous les quatre ans. Le contenu minimal du plan est le suivant: (i) diagnostic de la situation du saneamento básico et de son impact sur les conditions de vie, en incorporant des indicateurs sanitaires, épidémiologiques, environnementaux et socioéconomiques; (ii) des objectifs et cibles à court, moyen et long terme concernant les systèmes, visant l'universalisation de l'accès aux services (iii) des mesures de contingence et d'urgence (iv) des mécanismes et procédures d'évaluation de l'efficience et de I'efficacité des actions proposés. La loi détermine aussi que le plan doit être compatible avec d'autres plans municipaux et étatiques (le Schéma directeur de développement urbain et un éventuel plan de bassin hydrographique, l'équivalent du SAGE français).

Finalement, un autre instrument juridique permet de compléter et de préciser les orientations du Plan directeur de développement urbain : c'est la Loi municipale sur I'usage et l'occupation des sols, qui doit être, en principe, élaborée dans un délai fixé par le Plan. Toutefois, ce délai n'est pas toujours respecté ; un nombre important de municipalités ont approuvé leur Plan directeur mais ne disposent pas d'une loi l'usage et d'occupation des sols qui soit en accord avec les dispositions du Plan directeur. ${ }^{3}$ 


\section{Paris, Rio de Janeiro, la gestion des eaux pluviale et les instruments de planification}

Vu le nombre significatif des documents règlementaires sur la gestion des eaux pluviales ce texte sera limité à analyser certains d'entre eux, dans l'optique des principes du WSUD. On rappele que le WSUD concerne l'intégration du cycle de l'eau en ville en ayant comme objetctif assurer la sécurité des habitants face aux inondations, la qualité de vie et la protetion de l'environnement Dans le cas de Paris, deux instruments seront analysés: le Plan de Prévention des Risques d'Inondation du Département de Paris (PPRI) et le Projet de Zonage Sanitaire de la Ville de Paris. Dans le cas de Rio de Janeiro, il n'existe aucun instrument similaire au PPRI, c'est-à-dire un instrument ayant le pouvoir légal d'imposer des interdictions ou des restrictions à l'exercice des droits de construction et d'utilisation des sols. Ce qui est le plus proche, c'est le Schéma directeur de développement urbain, approuvé en 2011, qui peut imposer des restrictions à l'utilisation du sol, en prenant en compte les risques d'inondation.

Paris: vers une gestion durable des eaux pluviales? Les possibilités de mise en place des principes du WSUD

Le PPRI est un document réglementaire établi et approuvé par les services de l'Etat, en concertation avec la ville de Paris et avec les habitants, après débat public; il vise à caractériser le risque d'inondation résultant du débordement de la Seine et à recommander des mesures visant à réduire la vulnérabilité des biens et des personnes. Le PPRI porte sur l'ensemble du département de Paris. II a néanmoins été établi en concertation avec les départements limitrophes (les autres départements de la région île de France) pour que la définition de l'aléa soit cohérente d'un département à l'autre.

Il a un caractère normatif, définissant des interdictions ou des restrictions à l'exercice par les propriétaires de leur droit de construction et, en général, le droit d'occuper ou d'utiliser le terrain. Sa première version date de en 2003 et il a été révisé en 2007. La base du PPRI est établie à partir des plus hautes eaux connues (cette cote de référence est connue comme "PHEC") Dans le cas de Paris, ces plus hautes eaux correspondent aux niveaux des eaux atteints par la crue de janvier 1910. La version 2007 du règlement indique que dispositions contenues dans ce plan ne préjugent pas des règles plus restrictives, prises par le PLU ou par d'autres réglementations applicables sur le département de Paris ; mais que la révision ou la modification d'un document d'urbanisme ne pourra pas permettre d'instaurer des règles de construction plus permissives.

A partir de cette référence le zonage, établi dans la version 2003, a été conservé dans la version 2007. II comprend trois zones: (i) la zone verte correspond aux zones d'expansion des crues dont la vocation première est de permettre un stockage des eaux pour favoriser l'écrêtement de la crue, espace devant être laissé le plus libre possible de toute construction volumétrique; (ii) la zone rouge est la zone d'écoulement principal du fleuve en période de crue, devant être encombrée du moins d'obstacles possibles afin de permettre le libre écoulement des eaux; (iii) la zone bleue correspond aux zones urbanisées situées en zone inondable.

Dans la zone rouge se trouve le lit habituel du fleuve, mais aussi toutes les zones qui contribuent directement à son écoulement naturel, y compris les berges. Les 
installations, ouvrages, travaux et activités, permanents ou temporaires, présents sur les quais bas sont susceptibles de nuire au libre écoulement des eaux. Ils sont donc soumis à la réalisation d'une étude d'incidence qui doit porter notamment sur l'écoulement du fleuve. L'un des principes du WSUD est la fourniture, si possible, d'avantages esthétiques et paysagers des aménagements. Cela implique de repenser les installations et structures qui se trouvent sur les berges, pour améliorer la connexion des habitants à l'eau ; une connexion physique et visuelle. Dans le cadre du PPRI cet aspect, qui peut être travaillé dans une perspective de protection des berges, n'a pas été mentionné. Cependant, en 2012, la marie de Paris a mis en place et défendu un projet de reconquête des berges par rapport à la circulation automobile, qui permet d'accepter davantage de périodes d'inondation des quais bas.

La zone bleue comprend deux parties : une bleue sombre, qui correspond à des secteurs de bâti important exposé à des niveaux de submersion potentiellement supérieurs à un mètre, et une zone bleue clair correspondant au reste de la zone inondable, exclusion faite des zones verte et rouge.

Dans le cadre des principes de WSDU les zones inondables ne devraient pas être bâties. Cependant, en l'île-de-France, les PPRI ont vu le jour dans un contexte de forte urbanisation historique : $95 \%$ de la zone inondable en petite couronne est était déjà très urbanisée fin des années 1990. Cet instrument présente, donc, un certain nombre de limites (Faytre, 2017). Ces limites ont été reconnues dans la version 2003: "le département de Paris ne dispose que très ponctuellement de zones pouvant être réservées à l'expansion des crues et de zones où il est matériellement possible de restreindre les usages. Le PPRI visera donc à limiter l'augmentation du risque en limitant les extensions d'urbanisme et en contrôlant celles qui sont autorisées en zone inondable" (Direction de I'Urbanisme du Logement et de L'Équipement, 2003, p. 18)

Les prescriptions fixées pour la zone bleue ont pour objectifs : la réduction des activités pouvant présenter un risque pour l'environnement dans les zones d'alea le plus fort, et la prévention des dommages à l'environnement par l'intermédiaire des eaux du fleuve en crue ou de la nappe ; la réduction des risques en interdisant le stockage de biens sensibles ou coûteux dans les niveaux inondés, sauf à prendre des dispositions de protection particulières; la limitation de l'exposition directe à l'inondation des logements; pour les constructions neuves, l'obligation d'intégrer la connaissance du risque dans les techniques constructives et dans l'occupation des niveaux inondables.

En raison de l'importance supérieure du risque, les règles sont plus restrictives pour les logements inondables et pour les installations relevant de la législation sur la protection de l'environnement dans la zone bleu sombre. Pour aboutir aux objectifs de limitation des risques, il est notamment prévu de limiter partiellement la constructibilité autorisée dans Paris par rapport à la situation existante. Cependant, d'après Brun et Gache il y des brèches dans la réglementation. Les auteurs font référence à l'argument avancé par Nelly Olin, ministre chargé de l'Écologie en 2007, résumant la nouvelle doctrine de l'État en préambule d'un guide à l'usage des collectivités territoriales "la réduction de la vulnérabilité ne doit pas être systématiquement synonyme d'inconstructibilité ou de gel des territoires soumis aux risques" (Brun et Gache, 2013 p.4). En effet, le PPRI de 2003 va dans ce sens lorsqu'il mentionne que la limitation de construire, "appliquée à un centre urbain fortement urbanisé, ne doit néanmoins pas avoir pour conséquence 
de maintenir des espaces en friche ou en chantier au cœur de la capitale. II est en effet nécessaire de concilier les objectifs de prévention du risque d'inondation avec les contraintes de développement d'un centre urbain ancien. La constructibilité est donc admise sur uncertain nombre de secteurs stratégiques pour le développement économique ou social de Paris, ou d'intérêt national, limitativement identifies sur les cartes de zonages" (Direction de l'Urbanisme du Logement et de L'Équipement, 2003, p. 21-22).

Parmi ces zones est mentionné la a ZAC Paris Rive Gauche (125 ha, 13ème arr.), "dont l'achèvement présente un intérêt majeur tant pour le rééquilibrage est-ouest de Paris que pour l'installation d'équipements publics universitaires d'intérêt national" (Direction de I'Urbanisme du Logement et de L'Équipement, 2003, p.22).

Dans la version de 2003 du PPRI les 12ème, 13ème et 15ème arrondissements, sont ceux dont la constructibilité est admise car selon le plan ils présentent de nombreux enjeux pour le rééquilibrage en matière de diversités des fonctions urbaines et mixités sociales. Encore selon le plan, les secteurs stratégiques retenus pour ces arrondissements ont toutefois été limités pour concilier autant que possible les enjeux que présentent le développement de ces arrondissements avec l'objectif de prévention du risque d'inondationn. La versio 2007 du PPRI ajoute dispositions constructives garantissant la protection des biens et des personnes

La question est de savoir si les techniques proposées par le WSUD, c'est à dire les mesures préventives et compensatoires, sont effectivement mises en place par les aménageurs et si le résultat de ces mesures est évalué. Il est important que les mesures préventives soient prises en compte dès la conception des aménagements. Cela comprend : la réduction des surfaces imperméables, qui implique considérer la largeur des chaussées, la longueur des voies et des accès, la taille et la répartition des places de stationnement, la localisation des chemins piétonniers; I'augmentation du temps de concentration, obtenue par . la réduction de la vitesse de l'écoulement des eaux de pluie, qui peut se faire en diminuant les pentes et en augmentant la longueur du parcours de l'eau, en augmentant la rugosité des parois, en évitant de recourir aux caniveaux en dur par exemple. Les mesures compensatoires reposent sur deux principes: augmenter l'infiltration des eaux dans le sol pour réduire les volumes s'écoulant vers l'aval ; le stockage temporaire des eaux pour réguler les débits et réduire les vitesses d'écoulement. Parmi les aménagements favorisant ces mesures ont y trouve parmi d'autres: les toitures avec retenue temporaire des eaux pluviales, les toits verts, les noues et fossés, les bassins, les chaussées à structure réservoir; les tranchées et la récupération des eaux de pluie collectées sur les toits.

Cependant, la mise en place de ces aménagements dans les parcelles dépend des aménageurs publics et privés ; or, selon Brun et Gache, la réduction de la vulnérabilité aux inondations est, n'est pas prioritaire pour eux. Ce constat résulte d'une série d'entretiens menés en 2009 et 2010 auprès d'une vingtaine de professionnels de l'aménagement et de l'urbanisme qui exercent en région parisienne. Elle montre que les maîtres d'ouvrage publics et privés ne se saisissent pas encore de la question, en minimisent l'importance dans leurs cahiers des charges ou la cantonnent uniquement aux aspects techniques, voire réglementaires (Brun et Gache, 2010). 
En lle de France, ce n'est pas Paris mais les départements de petite couronne qui mènent les actions les plus remarquables. La Seine Saint Denis en particulier, qui a connu des épisodes d'inondations dues aux orages d'été dès les années 1970, a été pionnière dans la rétention et la gestion en temps réel des eaux de pluie : elle a d'abord organisé des bassins de stockage enterrés puis à ciel ouvert, partout où c'était possible dans la partie la plus dense et la plus proche de Paris. Dans la partie extérieure du département, la moindre densité a permis de réguler la gestion de la pluie en imposant des contraintes sur les permis de construire : stockage et réinfiltration de la pluie sur place, pas de permis de construire si ruissellement accru. Les nouveaux projets d'aménagement prévoient la mise en place de techniques paysagères de contrôle de la pluie dans les parcs et espaces extérieurs.

La ville de Paris a bien voté un plan général de verdissement des rues et des parcelles bâties (avec jardins sur les toits), mais sa mise en place pratique est lente car, contrairement au cas de Berlin, elle n'est pas encouragée financièrement ; de plus ce plan a été adopté davantage pour réduire le phénomène des îlots de chaleur s'accroissant avec le changement climatique, que pour ralentir les ruissellements. Les règles d'inconstructibilité en fond de parcelle, qui existaient depuis un siècle en tant que règle d'hygiène (aération traversante des immeubles), ont été supprimées dans le plan d'urbanisme des années 1980, ce qui conduit en fait à étendre les parkings souterrains sous toutes les parcelles et donc à réduire les possibilités d'infiltration de la pluie. Heureusement qu'il y a le réseau d'égouts visitable de Belgrand sous presque toutes les rues!

Cela dit, Paris dispose d'un autre instrument, le Plan Pluie, connu aussi comme zonage des eaux pluviales. II a été proposé par la mairie en 2013, en application d'une politique nationale ; il est passé par plusieurs étapes, et a finalement été adopté en 2018 (voté à I'unanimité au Conseil municipal le 22 mars 2018). Le plan a pour objectifs : la recherche par temps sec et humide pour l'optimisation du réseau de collecte et le transport des effluents vers les unités usine de traitement des eaux usées ; la réduction des rejets des systèmes unitaires dans la Seine lors des pluies normales pour améliorer la qualité de l'environnement naturel ; la réduction du risque de saturation et de débordement du réseau de drainage lors de fortes pluies dans certaines parties de Paris. Une partie de cet effort est conduite à l'échelle de l'agglomération par l'instance supra-locale publique qui gère les stations d'épuration et les plus gros collecteurs du grand Paris, le SIAAP (un des plus grands services d'assainissement au monde avec 8 millions d'usagers desservis). A cette échelle, plusieurs unités de prétraitement de la pollution pluviale ont été mises en place le long de la Seine et de la Marne, avec des tunnels de stockage comme celui d'Ivry - Massena.

Le plan Pluie de Paris est composé d'un zonage pluvial, d'un règlement et d'un guide technique d'application. Le zonage a identifié 348 micro bassins versants dans la ville pour lesquels ont été étudiées des pluies de périodes de retour allant d'un mois à un an, jusqu'à des pluies d'orage de dix et vingt ans. II a pris pour référence une pluie d'occurrence bisannuelle (16 mm en 24 heures), sachant que les pluies en dessous de $12 \mathrm{~mm}$ représentent plus de $80 \%$ des cas. Six zones correspondant au volume d'eau à abattre on été établies : jusqu'à $16 \mathrm{~mm}$ de pluie (correspondant à l'absorption de $100 \%$ de la pluie) dans les bois de Vincennes et de Boulogne ; et de $12 \mathrm{~mm}(80 \%$ d'absorption), $8 \mathrm{~mm}$ (55\%), $4 \mathrm{~mm}$ (30\%) dans Paris intra-muros, en fonction des 
caractéristiques du réseau d'assainissement et de la composition du sous-sol. La base du plan est d'augmenter l'infiltration des eaux de pluie, en evitant qu'elle arrive dans les réseaux publics. Pour cela il faut faire entrer la nature en ville et aménager des espaces publics favorisant l'infiltration : noues le long des trottoirs, toitures végétalisées, parking engazonnés, chaussées à structure réservoir, jardins de pluie.

L'État français a demandé que le zonage pluvial de Paris soit soumis à une évaluation environnementale en 2013. Cette évaluation est basée sur une étude d'impact hydraulique et une étude coût-bénéfice globale, incluant notamment des bénéfices écosystémiques qui ne sont pas forcément hydrauliques, comme par exemple la lutte contre les îlots de chaleur urbains, l'abattement des pollutions atmosphériques ou encore la sur-isolation thermique des toitures végétalisées. Le zonage pluvial devient alors une composante forte de la politique environnementale globale de la ville, dont il intègre des problématiques connexes. En étant approuvé en 2018 , il n'a pas encore produit des résultats.

Rio de Janeiro: les premiers pas vers une gestion durable des eaux pluviales, et les obstacles à surmonter

Le Plan directeur de développement urbain de Rio de Janeiro a été approuvé le 1er Février 2011 par la loi complémentaire $n^{\circ} 111$. Il conditionne les autres politiques et plans sectoriels. Ses objectifs seront poursuivis dans les plans pluriannuels gouvernementaux (PPAs) qui couvrent toujours une période de quatre ans, à compter de la deuxième année du mandat du chef de l'exécutif municipal et se terminant dans la première année du mandat suivant. En théorie, ce plan doit guider le fonctionnement des actions gouvernementales en le rendant plus efficace. Le plan pluriannuel doit rester compatible avec le Schéma directeur, qui est valable pour dix ans.

Les directives du Plan directeur de développement urbain doivent être intégrées dans les plans, programmes et projets de l'administration municipale directe et indirecte (les agences autonomes et les fondations) ainsi que dans les directives budgétaires.

En ce qui concerne la gestion des eaux pluviales et les risques d'inondation, le Plan directeur de Rio met un accent particulier sur la protection de zones fragiles ou vulnérables par rapport à l'occupation urbaine. II fournit une identification de ces zones dans les lignes directrices relatives à l'occupation urbaine: les pentes, les berges et les plaines inondables. Ces zones sont considérées impropres à la construction. Dans les terres basses, ,zones inondables, où l'on veut garder la perméabilité du sol, certains usages à faible impact, tels que l'agriculture, des parcs destinés à des activités de loisirs, et les zones résidentielles à faible densité, peuvent être autorisés, sous réserve des interventions appropriées pour permettre l'occupation et la construction en toute sécurité. Là la préservation de la vegetation native ou l'amenagement des parcs et la mise en place des techniques favorisant l'infiltration doivent jouer un rôle central.

En ce qui concerne les lotissements, le Plan directeur fixe comme limite au débit d'eau de pluie celui correspondant aux conditions préalables à la division en parcelles. Mais il ne rentre pas dans le détail technique, et laisse à la Loi d'usage et d'occupation du sol le soin de définir les dispositifs de contrôle d'augmentations de l'infiltration et le maintien des conditions pré-urbanisation. 
L'article 220 du Plan Directeur établit les directives pour la Politique de "Saneamento Básico" mais jusqu'à présent celle-ci n'a pas été traduite en loi. Parmi ces directives quelques unes se rapportent à la gestion des eaux pluviales: (i) concevoir, de manière intégrée et planifiée, les instruments de prévention, de minimisation et de gestion des inondations et glissements de terrain; (ii) récupérer la qualité et revaloriser les cours dieau pour en permettre une utilisation adéquate par des actions visant en priorité ceux présentant des situations à risque pour la vie des habitants (iii) contrôler les inondations à la source et définir les zones publiques pour les réserves foncières visant le contrôle des inondations.

L'article 226 liste les actions considérées comme structurantes liées à la gestion des eaux pluviales. Parmi ces actions, il est important de souligner : la mise en œuvre d'un schéma directeur de gestion des eaux pluviales; le contrôle des usages du sol et du développement urbain sur les berges inondables pour protéger la population des inondations et le milieu riverain ; le maintien d'une plus grande perméabilité des sols lors de l'octroi de permis de lotissements, obligeant ces derniers à prendre en compte les conditions topographiques pour le drainage naturel et les taux de perméabilité calculés pour le bassin versant ; la création d'un instrument juridique exigeant que les responsables de bâtiments publics et privés installent des systèmes de collecte des eaux pluviales, avec des dispositifs de réduction de la charge sur le réseau d'assainissement public ; la préservation des zones vertes urbaines y compris les forêts ; reboisement et réhabilitation des zones dégradées, en donnant priorité aux zones suivant : les lignes de drainage naturelles, en particulier les berges des rivières, les fonds des vallées et les plaines inondables ; la fixation réglementaire des utilisations du sol acceptables dans ces zones (parcs linéaires, zones de loisirs, jardins partagés), en accordant la priorité au maintien de la végétation d'origine ; la promotion d'actions et de campagnes permanentes d'information de la population sur la prévention des inondations ; le marquage physique des bandes non aedificandi par rapport aux risques d'inondation. On remarque dans les actions proposées un potentiel de préservation ou de reintroduction de la nature dans la ville.

Cependant, ces actions doivent être approfondies et détaillées dans la loi d'usage et occupation du sol. D'autres instruments d'urbanisme doivent aussi prendre en compte les actions structurantes concernant les eaux de pluie : la Loi de division du sol qui règlemente le découpage des nouveaux lotissements (avec ou sans voirie); et le Code des travaux et bâtiments qui établit les normes pour l'élaboration des projets de constructions et pour leur réalisation, de façon à garantir la préservation de la qualité du paysage de la ville et de ses bâtiments. II faut souligner que les deux derniers documents sont encore à l'état de projets de loi, et cela depuis 2013. Ce retard indique la difficulté des les approuver ; en effet les aménageurs, très fortement représentés au Conseil Municipal, y voient des obstacles à leurs intérêts. Le Sinduscom (Sindicato das Empresas da Construção Civil do Estado do Rio de Janeiro) qui est le représentant légal des entreprises du bâtiment de l'Etat de Rio de Janeiro et la ADEMI (Associação de Dirigentes de Empresas do Mercado Imobiliário) sont les plus actifs à faire pression au près des élus et à suivre ces projets de loi qu'ils considèrent prioritaires. Comme en France les maîtres d'ouvrage publics et privés ne se saisissent pas encore l'importance du projet pour la gestion des eaux de pluie. L'intérêt d'augmenter la surface construite et de réduire les coûts de construction prévaut. 
Les actions structurantes doivent aussi orienter le Schéma directeur des eaux pluviales, approuvé en tant que loi en décembre 2015, analysé ci-dessous. Ce plan affiche un objectif de gestion adéquate et durable des eaux pluviales, visant le contrôle des inondations dans la ville de Rio de Janeiro et assurant la santé publique, la sécurité de la vie et des biens publics et privés. Le document équivalent en France serait le PPRI.

Le Schéma directeur des eaux pluviales indique que les directives de zonage établies par le Plan de développement urbain encadrent les interventions proposées pour le macro drainage dans les bassins correspondants. Le territoire couvert par le Plan de développement urbain est divisé en quatre zones (i) zone contrôlée, où il existe de fortes restrictions sur la mise en œuvre de nouveaux projets résidentiels, commerciaux et de service ; (ii) zone d'occupation stimulée, où des incitations pour la revitalisation et la rénovation urbaine sont prévues, avec des investissements dans la construction d'infrastructures et de logements, ainsi que la réhabilitation de bâtiments ayant une importance historique, architecturale ou esthétique, et la stimulation des hôtels, commerces et services; (iii) zone d'occupation conditionnée où l'intensité constructive et l'installation d'activités économiques seront restreintes, ou conditionnées à capacité des réseaux d'infrastructure et subordonnée à la préservation de l'environnement et du paysage ; (iv) zone d'occupation assistée où la construction doit s'accompagner d'investissements publics dans les infrastructures et être conditionnée à la protection de l'environnement et à la préservation de l'activité agricole. II est important de noter que en France le rapport entre PLU et PPRI semble plus intégré que au Brésil

D'après les directives du Le Schéma directeur des eaux pluviales il est nécessaire d'inverser les effets néfastes de l'urbanisation dans les zones déjà construites et minimiser les impacts futurs de ce processus dans les zones en cours d'urbanisation. II souligne que dans les zones urbanisées, les mesures dites compensatoires pour la gestion des eaux de pluie seront inefficaces si les mesures structurelles nécessaires ne sont pas mises en œuvre dans l'ensemble du bassin hydrographique ; les mesures compensatoires restent, selon de plan, plus adaptées dans les zones en cours d'urbanisation. Cependant, le plan ne donne pas clairement une définition différenciant mesures structurelles et compensatoires. Dans le vocabulaire de la gestion des eaux pluviales utilisé au Brésil, on parle de mesures structurelles, correspondant à la modification des cours d'eau (les ouvrages hydrauliques, tels que les barrages, les digues et les canalisations), de mesures non structurelles utilisées pour faire face au cours d'eau dans les zones occupées (la délimitation des zones inondables, la restriction de l'utilisation et de l'occupation des terres, la mise en place de systèmes d'alerte) et de mesures compensatoires. Ces dernières sont celles qui cherchent à neutraliser les effets de l'urbanisation sur les processus hydrologiques, agissant dans la rétention et l'infiltration, avec des avantages pour la qualité du paysage et la préservation de l'environnement (Miguez, Verol e Rezende, 2016)

Il est évident que le Schéma directeur de Rio actuel met l'accent sur les mesures structurelles, telles que des interventions sur des cours d'eau canalisés (adéquation de la capacité hydraulique des canaux) et sur des réservoirs, souterrains et en superficie. Pour ces derniers, il n'y a aucun détail ou indication de les concevoir en tant que paysages multifonctionnels, ce qui est fondamental pour la qualité paysagère et aussi une possibilité de réintroduction de la nature en ville II n'a pas fait mention de techniques compensatoires, notamment à la parcelle (les noues et fosses, les chaussées à structure réservoir, les jardins filtrants, les tranchées et les conduites stockantes). 
La comparaison du Schéma directeur de Rio avec des plans de gestion des eaux pluviales d'autres villes brésiliennes, fait apparaitre une autre faiblesse : I'incomplétude de celui de Rio. II ne présente pas d'objectifs hiérarchisés à court, moyen et long terme, il ne définit pas de priorités, il ne fait pas une évaluation de l'organisation municipale pour la gestion des eaux pluviales, autant d'éléments qui sont pourtant fixés par la loi.

\section{Considerations finales}

La gestion d'eaux de pluie en ville a beacoup evolué au cours des dernières années vers une approche basée sur le respect le cycle urbain de l'eau, réconciliant la ville et les eux de pluie. Une fois maîtrisés les risques, ces eaux deviennent une ressource pour la ville. Le cadre règlementaire français concernant la gestion des eaux pluviales a évolué dans ce sens, avec des resultats en termes de la qualité du paysage dans certaines zones de l'agglomération parisienne, notamment dans le cadre de la reconquête des berges de la Seine à Paris et dans les Hauts de Seine, ainsi que dans les aménagements compensatoires en Seine Saint Denis. Mais, même si cette approche est actuellement obligatoire dans le cadre des règlements d'urbanisme, le rôle des maitres d'œuvre qui agissent sur les projets urbains reste décisif. Comme signalent Brun et Gache, ils sont certes plus sensibles au risque inondation que par le passé, mais ils disent ne pas être en capacité d'internaliser les coûts d'une reflexion innovante que le renouvellement urbain en zone inondable exige nécessairement (Brun et Gauche 2010).

Au Brésil le cadre réglementaire actuel de la gestion des eaux pluviales est certainement plus attentif à la durabilité. II incorpore certains principes de cette approche mais I'application de ce cadre depend de l'échelle muncipale, où il n'y a pas de régles fixant aux plans d'urbanisme des directives concernant la gestion des eaux pluviales. A Rio dans la gestion des eaux de pluie l'accent est encore mis sur les mesures hydrauliques traditionnelles. Les Plan directeur de developpement urbain intégre quelques principes d'une gestion durable des eaux pluviales, mais les règlements d'urbanisme restent inachevés.

Le Schéma directeur des eaux pluviales de Rio n'est pas vraiment conforme aux principes de WSUD qui valorisent les techniques compensatoires même dans les zones urbanisées. Les actions menées en Seine Saint Denis, montrent qu'elles peuvent être déployées dans des zones déjà urbanisées en utilisant des stockages à ciel ouvert, visibles et intégrés au tissu urbain. L'eau pluviale devient, ainsi, un élément de valorisation du projet urbain, possédant une diversité de fonctions et d'usages.

C'est le cas aussi du Plan de Pluie parisien, qui prend en compte l'échelle de la parcelle, au delà de ce que propose le Schéma directeur de Rio. En effet, dans la perspective du WSUD la multiplication de ces techniques compensatoires, dans l'ensemble d'un bassin hydrographique, en travaillant à plusieurs échelles, peut réduire la nécessité d'installer de grandes structures pour retenir l'excès d'eau pendant les périodes pluvieuses, tels que les réservoirs ouverts et souterrains priorisés dans le schéma directeur de Rio. Au même temps, à partir de ces techniques on peut envisager les eaux de pluie comme un atout potentiel de développement urbain intégré avec la nature. 


\section{Références bibliographiques}

BARRAQUÉ B.; ISNARD, L.; Souriau J., 2015. How Water Services Manage Territories and Technologies: History and Current Trends in Developed Countries, in Grafton, Q., Daniell, K.A., Nauges, C., Rinaudo, J.-D., Chan, N.W.W. (Eds.) Understanding and Managing Urban Water in Transition, Global Issues in Water Policy n¹5, Springer, ch.2, pp 33-59.

BRITTO, A. L. N. P., 2011. O saneamento ambiental nos planos diretores municipais. In: Santos Junior, O. A., Montandon, D. T. (dir.). Os planos Diretores Municipais Pós-estatuto das Cidades : balanço critico e perspectivas. Rio de Janeiro, Letra Capital Observatório das Metrópoles, p. 127-153.

BRUN, A. et Gache, F., 2013. Risque inondation dans le Grand Paris: la résilience estelle un concept opératoire ?, VertigO - la revue électronique en sciences de l'environnement [En ligne], Regards / Terrain, consulté le 06 avril 2018. URL <http://journals.openedition.org/ vertigo/14339> ; DOI : 10.4000/vertigo.14339.

BRUN, A. et F. Gache, 2010. L'aménagement des zones inondables en ÎledeFrance. Regards croisés de praticiens, IIBRBS-Grands Lacs de Seine, Online <http://seinegrandslacs.fr/sites/ default/files/2010-regards-croises-de-patriciens-amenagement-des-zones-inondables.pdf>.

CHATZIS K. \& DUPUY G., 2000. How to dispense with empiricism: The 'Caquot formula' and post-war drainage policy in France, Water Policy, 2000-2, 267-281.

CHOCAT B., 2008. Etat de l'art sur la gestion urbaine des eaux pluviales et leur valorisation -Tendances d'évolution et technologies en développement, publication conjointe de l'Office International de l'Eau et de l'Office National de l'Eau et des Milieux Aquatiques, Juillet.

FAYTRE, L., 2017. Témoignage d'acteurs - Urbanisme et risque "inondation", le cas de l'îlede-France, [online], Revue Gestion du risque inondation: connaissances et outils au service de l'aménagement des territoires, n²3, p. 8-11.

FLETCHER, T. D.; SHUSTER, W.; HUNT, W.F.; ASHLEY, F.; BUTLER, D.; SCOTT, A.; TROWSDALE, S.; BARRAUD, S.; SEMADENI-DAVIES, A; BERTRAND-KRAJEWSKI, J; MIKKELSEN, P.T.; RIVARD, G.; UHL, M; DAGENAIS, D. \& M.V., 2015. SUDS, LID, BMPs, WSUD and more The evolution and application of terminology surrounding urban drainage, Urban Water Journal, 12:7, 525-542.

LANGENBACH, H.; ECKART, J. \& SCHRöDER, G,. Water Sensitive Urban Design - results and principles, in: Proceedings of 3rd SWITCH Scientific Meeting, 2008 Belo Horizonte, Brazil.

HOYER, J.; DICKHAUT, W.; KRONAWITTER, L. \& Weber, B., 2011. Water Sensitive Urban Design - Principles and Inspiration for Sustainable Stormwater Management in the City of the Future. Germany, HafenCity Universität Hamburg (HCU).

MIGUEZ, M.G; VÉROL, A. P.; REZENDE, O. M., 2016. Drenagem Urbana do projeto tradicional à sustentabilidade. Rio de Janeiro, Elsevier.

PLAN URBAIN (J.M. Lelong, dir.), 1995. L'eau dans la ville, 1983-1994, bilan général, Presses de l'Ecole Nationale des Ponts et Chaussées.

SANTOS JUNIOR, O A.; MONTADON, D. T., 2011. Síntese, Desafios e Recomendações. In: Orlando Alves dos Santos Junior, Daniel Todtmann Montandon. (Org.). Os Planos Diretores Municipais Pós-Estatuto das Cidades: balanço crítico e perspectivas. 1ed. Rio de Janeiro, Letra Capital, p. 27-56.

SIAAP (Syndicat Interdépartemental pour l'Assainissement de I'Agglomération Parisienne) et Observatoire des Usagers de I'Assainissement, 2006. La pluie en ville, nuisance ou ressource, actes du colloque du 16 mai 2006. <https://www.oieau.fr/eaudoc/system/files/ documents/42/210415/210415_doc.pdf>.

WONG, T.H.F., 2007. Water sensitive urban design; the journey thus far. Australian Journal of Water Resources, 110 (3), 213-222. 